\title{
COUNTING THE NUMBER OF CENTRALIZERS OF 2-ELEMENT SUBSETS IN A FINITE GROUP
}

\author{
A. R. ASHRAFI ${ }^{\star}$, F. KOOREPAZAN-MOFTAKHAR AND M. A. SALAHSHOUR
}

\begin{abstract}
Suppose $G$ is a finite group. The set of all centralizers of 2 -element subsets of $G$ is denoted by $2-\operatorname{Cent}(G)$. A group $G$ is called $(2, n)$-centralizer if $|2-\operatorname{Cent}(G)|=n$ and primitive $(2, n)$-centralizer if $|2-\operatorname{Cent}(G)|=\left|2-\operatorname{Cent}\left(\frac{G}{Z(G)}\right)\right|=n$, where $Z(G)$ denotes the center of $G$. The aim of this paper is to present the main properties of $(2, n)$-centralizer groups among them a characterization of $(2, n)$-centralizer and primitive $(2, n)$-centralizer groups, $n \leq 9$, are given.
\end{abstract}

Keywords: $(2, n)$-centralizer group, primitive $(2, n)$-centralizer group, $n$-centralizer group.

2010 Mathematics Subject Classification: Primary: 20C15; Secondary: 20D15.

\section{INTRODUCTION}

Throughout this paper all groups are assumed to be finite and for a subset $A$ of a group $G$, the centralizer subgroup of $A$ in $G$ is denoted by $C_{G}(A)$. The cyclic group of order $n$, the dihedral group of order $2 n$, the alternating group on $n$ symbols and the symmetric group of degree $n$ are denoted by $\mathbb{Z}_{n}, D_{2 n}, A_{n}$ and $S_{n}$, respectively. The holomorph of $G$ is denoted by $\operatorname{Hol}(G)$ and $Z(G)$ denotes the center of $G$. The second center of $G$ which is denoted by $Z_{2}(G)$, is defined as $\frac{Z_{2}(G)}{Z(G)}=Z\left(\frac{G}{Z(G)}\right)$. Set:

$$
\begin{aligned}
\operatorname{Cent}(G) & =\left\{C_{G}(x) \mid x \in G\right\}, \\
2-\operatorname{Cent}(G) & =\left\{C_{G}(\{x, y\}) \mid x, y \in G \& x \neq y\right\} .
\end{aligned}
$$

The group $G$ is called $n$-centralizer if $|\operatorname{Cent}(G)|=n$ and in addition, if $\frac{G}{Z(G)}$ is an $n$-centralizer group, then $G$ is said to be primitive $n$-centralizer group. It is called $(2, n)$-centralizer if $|2-C e n t(G)|=n$ and primitive $(2, n)$-centralizer if $|2-\operatorname{Cent}(G)|=\left|2-\operatorname{Cent}\left(\frac{G}{Z(G)}\right)\right|=n$. To simplify our argument, a subgroup of $G$ in the form $C_{G}(\{x, y\})$ is called a 2 -element centralizer for $G$. It is clear that the number of $2-$ element centralizers of $G$ is equal to $|2-\operatorname{Cent}(G)|$. Note that for each element $x, y \in G$, $C_{G}(\{x, y\})=C_{G}(x) \cap C_{G}(y)$.

The study of finite groups in terms of $|\operatorname{Cent}(G)|$ was started by Belcastro and Sherman in 1994 [7. It is easy to see that a group is 1 -centralizer if and only if it is abelian and there is no 2and 3-centralizer group. One of the present authors (ARA) [2] constructed $n$-centralizer groups, for each $n \neq 2,3$ and primitive $n$-centralizer groups for each odd positive integer $\neq 3$. In [4], the authors proved that a group with $\leq 21$ element centralizers is solvable and gave a characterization of the simple group $A_{5}$ according to the number of element centralizers. Zarrin [20] presented another proof for solvability of groups with at most 21 element centralizers. Zarrin [19] computed $|C e n t(G)|$ for all minimal simple groups. As a consequence he proved that there are non-isomorphic finite simple

${ }^{\star}$ Corresponding author (Email: ashrafi@kashanu.ac.ir). 
groups $G$ and $H$ such that $|\operatorname{Cent}(G)|=|\operatorname{Cent}(H)|$. Kitture [14] proved that isoclinic groups have the same number of element centralizers and for each positive integer $n$ different from 2 and 3 , there are only finitely many groups, up to isoclinism, with exactly $n$ element centralizers.

Suppose $R$ denotes the semidirect product of a cyclic group of order 5 by a cyclic group of order 4 acting faithfully. It is easy to see that $R$ can be presented as $R=\left\langle x, y \mid x^{5}=y^{4}=1, x y=y x^{3}\right\rangle$. A finite $n$-centralizer group $G$ with $n \leq 10$ were determined in terms of the structure of $\frac{G}{Z(G)}$. For the sake of completeness, we collect these results in the following theorems:

Theorem 1.1. Suppose $G$ is a finite group. Then,

(1) $G$ is 4 -centralizer if and only if $\frac{G}{Z(G)} \cong \mathbb{Z}_{2} \times \mathbb{Z}_{2}$ [7].

(2) $G$ is 5-centralizer if and only if $\frac{G}{Z(G)} \cong \mathbb{Z}_{3} \times \mathbb{Z}_{3}$ or $S_{3}$ [7].

(3) If $G$ is 6-centralizer, then $\frac{G}{Z(G)} \cong \mathbb{Z}_{2} \times \mathbb{Z}_{2} \times \mathbb{Z}_{2}, \mathbb{Z}_{2} \times \mathbb{Z}_{2} \times \mathbb{Z}_{2} \times \mathbb{Z}_{2}$, D8 or $A_{4}$ [2].

(4) $G$ is primitive 7 -centralizer if and only if $\frac{G}{Z(G)} \cong D_{10}$ or $R[3]$.

(5) $G$ is 7 -centralizer if and only if $\frac{G}{Z(G)} \cong \mathbb{Z}_{5} \times \mathbb{Z}_{5}, D_{10}$ or $R$ [1].

(6) If $G$ is 8 -centralizer, then $\frac{G}{Z(G)} \cong \mathbb{Z}_{2} \times \mathbb{Z}_{2} \times \mathbb{Z}_{2}$, D $D_{12}$ or $A_{4}$ [1].

(7) $G$ is 9 -centralizer if and only if $\frac{G}{Z(G)} \cong D_{14}, \mathbb{Z}_{7} \times \mathbb{Z}_{7}, H$ ol $\left(\mathbb{Z}_{7}\right)$ or a non-abelian group of order 21 [13].

(8) $G$ is primitive 9 -centralizer if and only if $\frac{G}{Z(G)} \cong D_{14}, H o l\left(\mathbb{Z}_{7}\right)$ or a non-abelian group of order 21 [5].

(9) There is no 10-centralizer groups of odd order [10].

(10) If $G$ is a primitive 11 - centralizer group of odd order, then $\frac{G}{Z(G)} \cong\left(\mathbb{Z}_{9} \times \mathbb{Z}_{3}\right) \rtimes \mathbb{Z}_{3}[15]$.

A group in which every non-central element has an abelian centralizer is called a $C A$-group [17].

Theorem 1.2. Suppose $n$ is a positive integer and $p$ is a prime. Then we have:

(1) ([1, Lemma 2.4.]) Let $G$ be a finite non-abelian group and $\left\{x_{1}, \ldots, x_{r}\right\}$ be a set of pairwise non-commuting elements of $G$ with maximal size. Then

(a) $r \geq 3$.

(b) $r+1 \leq|\operatorname{Cent}(G)|$.

(c) $r=3$ if and only if $|\operatorname{Cent}(G)|=4$.

(d) $r=4$ if and only if $|\operatorname{Cent}(G)|=5$.

(2) ([1, Proposition 2.5]) Let $G$ be a finite group and let $X=\left\{x_{1}, \ldots, x_{r}\right\}$ be a set of pairwise non-commuting elements of $G$ having maximal size.

(a) If $|\operatorname{Cent}(G)|<r+4$, then for each element $x \in G, C_{G}(x)$ is abelian if and only if $C_{G}(x)=C_{G}\left(x_{i}\right)$ for some $i \in\{1, \ldots, r\}$.

(b) If $|\operatorname{Cent}(G)|=r+2$, then there exists a proper non-abelian centralizer $C_{G}(x)$ which contains $C_{G}\left(x_{i_{1}}\right), C_{G}\left(x_{i_{2}}\right)$ and $C_{G}\left(x_{i_{3}}\right)$ for three distinct $i_{1}, i_{2}, i_{3} \in\{1, \ldots, r\}$.

(3) ([1, Lemma 2.6]) Let $G$ be a finite non-abelian group. Then every proper centralizer of $G$ is abelian if and only if $|\operatorname{Cent}(G)|=r+1$, where $r$ is the maximal size of a set of pairwise non-commuting elements of $G$.

(4) ([4, Theorem 1]) If $G$ is a finite group and $\frac{G}{Z(G)} \cong A_{5}$, then $|\operatorname{Cent}(G)|=22$ or 32 .

(5) ([4, Theorem 2]) If $G$ is a finite simple group and $\mid$ Cent $(G) \mid=22$, then $G \cong A_{5}$. 
(6) ([6, Lemma 2.1]) Let $\left|\frac{G}{Z(G)}\right|=p q r$, where $p, q$ and $r$ are primes not necessarily distinct. Then $G$ is $C A$-group.

(7) ([6, Proposition 2.2]) Let $p$ be the smallest prime dividing $|G|$. If $[G: Z(G)]=p^{3}$, then $|\operatorname{Cent}(G)|=p^{2}+p+2$ or $p^{2}+2$.

(8) ([6, Theorem 2.3]) If $G$ has an abelian normal subgroup of prime index, then $|C e n t(G)|=$ $\left|G^{\prime}\right|+2$.

(9) ([6, Proposition 2.8]) Let $\frac{G}{Z(G)}$ be non-abelian, $n$ be an integer and $p$ be a prime. If $\frac{G}{Z(G)} \cong$ $\mathbb{Z}_{n} \rtimes \mathbb{Z}_{p}$, then $G$ has an abelian normal subgroup of index $p$ and $\left|G^{\prime}\right|=n$.

(10) ([6, Proposition 2.9]) Let $\frac{G}{Z(G)}$ be non-abelian. If $\frac{G}{Z(G)} \cong \mathbb{Z}_{n} \rtimes \mathbb{Z}_{p}$, then $|\operatorname{Cent}(G)|=n+2$.

(11) ([6, Lemma 2.10]) If $\frac{G}{Z(G)}$ is non-abelian and $\frac{G}{Z(G)} \cong \mathbb{Z}_{n} \rtimes \mathbb{Z}_{p}$ then $G$ is a $C A$-group.

(12) $\left(\left[7\right.\right.$, Theorem 5]) Let $p$ ba a prime. If $\frac{G}{Z(G)} \cong \mathbb{Z}_{p} \times \mathbb{Z}_{p}$, then $|\operatorname{Cent}(G)|=p+2$.

(13) ([8, Lemma 4, p. 303]) If $H$ is a normal abelian subgroup of a non-abelian group $G$ of prime index $p$, then $|G|=p\left|G^{\prime}\right||Z(G)|$, and $\left|G: C_{G}(x)\right|=\left|G^{\prime}\right|$ for $x \in G-H$.

(14) ([9, Theorem A (I)]) Let $G$ be a non-abelian group. Then $G$ is a $C A$-group if and only if $G$ has an abelian normal subgroup of prime index.

(15) ([12, Proposition 1.2]) If $G$ is a $C A$-group, then for each non-central element $x$ and $y, C_{G}(x)=$ $C_{G}(y)$ or $C_{G}(x) \cap C_{G}(y)=Z(G)$.

(16) (4] and [20, Theorem A]) Let $G$ be an $n$-centralizer finite group with $n \leq 21$, then $G$ is soluble.

Throughout this paper our notations are standard and taken mainly from [16]. Our calculations are done with the aid of GAP [18].

\section{Some Basic Properties of $(2, n)$-Centralizer Groups}

In this section, a characterization of finite groups with at most nine 2-element centralizers are given.

Lemma 2.1. Let $G$ be a non-abelian group. Then

(1) $Z(G) \notin \operatorname{Cent}(G)$.

(2) $G \notin 2-\operatorname{Cent}(G)$ if and only if $Z(G)=1$.

Proof. Let $Z(G) \in \operatorname{Cent}(G)$. Set $\operatorname{Cent}(G)=\left\{G, C_{G}\left(x_{1}\right), \ldots, C_{G}\left(x_{r}\right)\right\}$, where $x_{i} \notin Z(G), 1 \leq i \leq r$. Then either $Z(G)=G$ or there exists $x_{i}, 1 \leq i \leq r$, such that $Z(G)=C_{G}\left(x_{i}\right)$. Both of these cases will lead to contradiction. Thus, $Z(G) \notin \operatorname{Cent}(G)$ that completes Part (1). To prove the Part (2), it is enough to note that for every $x, y \in G$ such that $x \neq y, x, y \in Z(G)$ if and only if $C_{G}(x) \cap C_{G}(y)=G$.

The previous lemma shows that if $Z(G) \in \operatorname{Cent}(G)$, then $G$ is abelian. The following simple lemmas are crucial in our main results.

Lemma 2.2. Let $G$ be a non-abelian group. Then

(1) If $Z(G) \neq 1$, then $\operatorname{Cent}(G) \varsubsetneqq 2-\operatorname{Cent}(G)$. In particular $|C e n t(G)|<|2-\operatorname{Cent}(G)|$.

(2) If $Z(G)=1$, then $|\operatorname{Cent}(G)| \leq|2-\operatorname{Cent}(G)|$.

Proof. Our main proof will consider the following two separate cases: 
(1) Let $Z(G) \neq 1$ and $\operatorname{Cent}(G)=\left\{G, C_{G}\left(x_{1}\right), \ldots, C_{G}\left(x_{r}\right)\right\}$ in which $x_{i} \notin Z(G), 1 \leq i \leq r$. Since $Z(G) \neq 1$, by Lemma $2.1(2), G \in 2-\operatorname{Cent}(G)$. On the other hand, for every $1 \leq$ $i \leq r, C_{G}\left(x_{i}\right)=C_{G}\left(\left\{x_{i}, e\right\}\right) \in 2-\operatorname{Cent}(G)$. Therefore, $\operatorname{Cent}(G) \subseteq 2-\operatorname{Cent}(G)$. Suppose $2-\operatorname{Cent}(G)=\operatorname{Cent}(G)$. Then for every $x, y \in G \backslash Z(G)$

$$
C_{G}(x) \cap C_{G}(y)=C_{G}(\{x, y\}) \in 2-\operatorname{Cent}(G)=\operatorname{Cent}(G)=\left\{G, C_{G}\left(x_{1}\right), \ldots, C_{G}\left(x_{r}\right)\right\}
$$

and by a similar argument as the proof of Lemma $2.1(2), C_{G}(x) \cap C_{G}(y) \neq G$. So there exists $1 \leq i \leq r$, such that $C_{G}(x) \cap C_{G}(y)=C_{G}\left(x_{i}\right)$. By our assumption, $Z(G)=C_{G}\left(x_{1}\right) \cap C_{G}\left(x_{2}\right) \cap$ $\ldots \cap C_{G}\left(x_{r}\right)$. Now an inductive argument shows that $Z(G)=C_{G}\left(x_{t}\right)$, for some $t, 1 \leq t \leq r$. Therefore, $x_{t} \in Z(G)$ which is impossible.

(2) Suppose $Z(G)=1$ and set $C e n t(G)=\left\{G, C_{G}\left(x_{1}\right), \ldots, C_{G}\left(x_{r}\right)\right\}$ in which $x_{i} \notin Z(G), 1 \leq i \leq r$. By Lemma 2.1(2), $G \notin 2-\operatorname{Cent}(G)$ and according to Lemma $2.2(1), \operatorname{Cent}(G) \backslash\{G\} \subset$ $2-\operatorname{Cent}(G)$. Thus $|\operatorname{Cent}(G)| \leq|2-\operatorname{Cent}(G)|$.

This completes the proof.

Lemma 2.3. Suppose $H$ and $K$ are two finite groups. Then $|\operatorname{Cent}(H \times K)|=|\operatorname{Cent}(H)||\operatorname{Cent}(K)|$. In particular, if $H$ is abelian, then $|\operatorname{Cent}(H \times K)|=|\operatorname{Cent}(K)|$.

Proof. It is clear that if $(x, y) \in H \times K$, then $C_{H \times K}((x, y))=C_{H}(x) \times C_{K}(y)$. We now consider four separate cases for the pair $(x, y)$ as follows:

(1) $x$ is a central element of $H$ and $y$ is a central element of $K$. In this case, $C_{H \times K}((x, y))=$ $C_{H}(x) \times C_{K}(y)=H \times K$. Therefore in this case we have only one element centralizer in $H \times K$.

(2) $x$ is not central in $H$ but $y$ is a central element of $K$. Since $C_{H \times K}((x, y))=C_{H}(x) \times K$, there are exactly $|\operatorname{Cent}(H)|-1$ element centralizers different from $H \times K$.

(3) $x$ is a central element of $H$ and $y$ is not central in $K$. In this case, $C_{H \times K}((x, y))=H \times C_{K}(y)$ and again we have $|\operatorname{Cent}(K)|-1$ element centralizers different from $H \times K$. Note that all of these element centralizers are different from those given in part (2).

(4) $x$ is not central in $H$ and $y$ is not central in $K$. By our assumption, $C_{H \times K}((x, y))=C_{H}(x) \times$ $C_{K}(y)$ and there are $(|\operatorname{Cent}(H)|-1)(|\operatorname{Cent}(K)|-1)$ different element centralizers in $H \times K$.

Therefore

$$
\begin{aligned}
|\operatorname{Cent}(H \times K)| & =1+(|\operatorname{Cent}(H)|-1)+(|\operatorname{Cent}(K)|-1)+(|\operatorname{Cent}(H)|-1))(|\operatorname{Cent}(K)|-1) \\
& =|\operatorname{Cent}(H)||\operatorname{Cent}(K)| .
\end{aligned}
$$

The second part is obvious.

Theorem 2.4. Suppose $H$ and $K$ are two finite groups. Then

$$
|2-\operatorname{Cent}(H \times K)|=|2-C e n t(H)||2-C e n t(K)|+\delta(K)|2-C e n t(H)|+\delta(H)|2-C e n t(K)|
$$

in which

$$
\delta(G)=\left\{\begin{array}{cc}
1 & Z(G)=1 \\
0 & Z(G) \neq 1
\end{array}\right.
$$


Proof. It is clear that for every $(x, y) \in H \times K, C_{H \times K}((x, y))=C_{H}(x) \times C_{K}(y)$. For every $(m, n) \in$ $H \times K,(m, n) \in C_{H \times K}((x, y)) \cap C_{H \times K}((a, b))$ if and only if $(m, n) \in C_{H \times K}((x, y))$ and $(m, n) \in$ $C_{H \times K}((a, b))$ if and only if $(m, n) \in\left(C_{H}(x) \times C_{K}(y)\right)$ and $(m, n) \in\left(C_{H}(a) \times C_{K}(b)\right)$ if and only if $m \in C_{H}(x), n \in C_{K}(y), m \in C_{H}(a)$ and $n \in C_{K}(b)$ if and only if $m \in C_{H}(x) \cap C_{H}(a)$ and $n \in C_{K}(y) \cap C_{K}(b)$ if and only if $(m, n) \in\left(C_{H}(x) \cap C_{H}(a)\right) \times\left(C_{K}(y) \cap C_{K}(b)\right)$. Therefore, for every $(x, y),(a, b) \in H \times K$,

$$
C_{H \times K}((x, y)) \cap C_{H \times K}((a, b))=\left(C_{H}(x) \cap C_{H}(a)\right) \times\left(C_{K}(y) \cap C_{K}(b)\right) .
$$

We have the following four cases:

(1) $Z(H) \neq 1$ and $Z(K) \neq 1$. By Lemma 2.2(1), Cent $(H) \subset 2-\operatorname{Cent}(H)$ and $C e n t(K) \subset$ $2-\operatorname{Cent}(K)$. Now by Equation 2.1. $|2-\operatorname{Cent}(H \times K)|=|2-\operatorname{Cent}(H)||2-\operatorname{Cent}(K)|$.

(2) $Z(H) \neq 1$ and $Z(K)=1$. Again apply Lemma 2.2(1) to show that $\operatorname{Cent}(H) \subset 2-C e n t(H)$ and by Lemma $2.1(2) K \in \operatorname{Cent}(K) \backslash 2-\operatorname{Cent}(K)$. Therefore,

$$
|2-\operatorname{Cent}(H \times K)|=|2-\operatorname{Cent}(H)||2-\operatorname{Cent}(K)|+|2-\operatorname{Cent}(H)| .
$$

(3) $Z(H)=1$ and $Z(K) \neq 1$. A similar argument as Part (2) show that

$$
|2-\operatorname{Cent}(H \times K)|=|2-\operatorname{Cent}(H)||2-\operatorname{Cent}(K)|+|2-\operatorname{Cent}(K)| \text {. }
$$

(4) $Z(H)=1$ and $Z(K)=1$. Again as Part $(2),|2-C e n t(H \times K)|=|2-C e n t(H)||2-C e n t(K)|+$ $|2-\operatorname{Cent}(H)|+|2-\operatorname{Cent}(K)|$.

Therefore,

$$
|2-\operatorname{Cent}(H \times K)|=|2-\operatorname{Cent}(H)||2-\operatorname{Cent}(K)|+\delta(K)|2-C e n t(H)|+\delta(H)|2-C e n t(K)|,
$$

which completes the proof.

Example 2.5. In this example the number of 2-element subset centralizers of the group $S_{n} \times S_{n}$ is computed. Suppose $S_{n}$ has exactly $r$ 2-element subset centralizers. Since $S_{n}$ is centerless,

$$
\left|2-\operatorname{Cent}\left(S_{n} \times S_{n}\right)\right|=\left|2-\operatorname{Cent}\left(S_{n}\right)\right|^{2}+2\left|2-\operatorname{Cent}\left(S_{n}\right)\right|=r^{2}+2 r .
$$

Corollary 2.6. Suppose $H_{1}, H_{2}, \ldots, H_{n}$ are groups and $A=\{1,2, \ldots, n\}$. Then,

$$
\begin{aligned}
\left|2-\operatorname{Cent}\left(\prod_{i=1}^{n} H_{i}\right)\right| & =\prod_{i=1}^{n}\left|2-\operatorname{Cent}\left(H_{i}\right)\right| \\
& +\sum_{i=1}^{n-1}\left(\sum_{B_{i} \subset A}\left(\left(\prod_{x \in B_{i}} \delta\left(H_{x}\right)\right)\left(\prod_{y \in B_{i}^{\prime}}\left|2-\operatorname{Cent}\left(H_{y}\right)\right|\right)\right)\right)
\end{aligned}
$$

in which $B_{i}$ is a $i$-subset of $A$ and $B_{i}^{\prime}=A-B_{i}$. Moreover, by means of $\sum_{i=1}^{n-1} \sum_{B_{i} \subset A}$ we consider summation on all $i$-element subsets of $A$ such that $1 \leq i \leq n-1$.

Proof. Induct on $n$. By Theorem 2.4, the result is valid for $n=2$. Suppose the result is correct for $n=k, k \geq 3$. Then,

$$
\left|2-\operatorname{Cent}\left(\prod_{i=1}^{k} H_{i}\right)\right|=\prod_{i=1}^{k}\left|2-\operatorname{Cent}\left(H_{i}\right)\right|+M_{k-1}
$$


in which

$$
M_{k-1}=\sum_{i=1}^{k-1}\left(\sum_{B_{i} \subset A}\left[\left(\prod_{i \in B_{i}} \delta\left(H_{i}\right)\right)\left(\prod_{i \in B_{i}^{\prime}}\left|2-\operatorname{Cent}\left(H_{i}\right)\right|\right)\right]\right) .
$$

We prove the result for $n=k+1$. Since $\delta\left(\prod_{i=1}^{n} H_{i}\right)=\prod_{i=1}^{n} \delta\left(H_{i}\right)$, by Theorem 2.4

$$
\begin{aligned}
\left|2-\operatorname{Cent}\left(\prod_{i=1}^{k+1} H_{i}\right)\right|= & \left|2-\operatorname{Cent}\left(\prod_{i=1}^{k} H_{i} \times H_{k+1}\right)\right| \\
= & \left|2-\operatorname{Cent}\left(\prod_{i=1}^{k} H_{i}\right)\right|\left|2-\operatorname{Cent}\left(H_{k+1}\right)\right| \\
& +\delta\left(H_{k+1}\right)\left|2-\operatorname{Cent}\left(\prod_{i=1}^{k} H_{i}\right)\right|+\delta\left(\prod_{i=1}^{k} H_{i}\right)\left|2-\operatorname{Cent}\left(H_{k+1}\right)\right| \\
= & \left(\prod_{i=1}^{k}\left|2-\operatorname{Cent}\left(H_{i}\right)\right|+M_{k-1}\right)\left|2-\operatorname{Cent}\left(H_{k+1}\right)\right| \\
& +\delta\left(H_{k+1}\right)\left(\prod_{i=1}^{k}\left|2-\operatorname{Cent}\left(H_{i}\right)\right|+M_{k-1}\right) \\
& +\left(\prod_{i=1}^{n} \delta\left(H_{i}\right)\right)\left|2-C e n t\left(H_{k+1}\right)\right| \\
= & \left(\prod_{i=1}^{k}\left|2-\operatorname{Cent}\left(H_{i}\right)\right|\right)\left|2-\operatorname{Cent}\left(H_{k+1}\right)\right| \\
& +M_{k-1}\left|2-\operatorname{Cent}\left(H_{k+1}\right)\right|+\delta\left(H_{k+1}\right)\left(\prod_{i=1}^{k}\left|2-C e n t\left(H_{i}\right)\right|\right) \\
& +\delta\left(H_{k+1}\right) M_{k-1}+\left(\prod_{i=1}^{n} \delta\left(H_{i}\right)\right)\left|2-C e n t\left(H_{k+1}\right)\right| \\
= & \prod_{i=1}^{k+1}\left|2-\operatorname{Cent}\left(H_{i}\right)\right|+M_{k}, \\
& \\
& \\
& \\
& \\
&
\end{aligned}
$$

proving the corollary.

Example 2.7. In this example the case of $n=3$ in Corollary 2.6 is completed. Suppose $A=\{1,2,3\}$ and for $i=1,2, B_{1}=\{1\},\{2\}$ or $\{3\}$ and $B_{2}=\{1,2\},\{1,3\}$ or $\{2,3\}$. Therefore,

$$
\begin{aligned}
\left|2-\operatorname{Cent}\left(H_{1} \times H_{2} \times H_{3}\right)\right| & =\left|2-C \operatorname{ent}\left(H_{1}\right)\right|\left|2-C e n t\left(H_{2}\right)\right|\left|2-C \operatorname{ent}\left(H_{3}\right)\right| \\
& +\delta\left(H_{1}\right)\left|2-C \operatorname{ent}\left(H_{2}\right)\right|\left|2-C \operatorname{Cent}\left(H_{3}\right)\right| \\
& +\delta\left(H_{2}\right)\left|2-C \operatorname{ent}\left(H_{1}\right)\right|\left|2-C \operatorname{Cent}\left(H_{3}\right)\right| \\
& +\delta\left(H_{3}\right)\left|2-C \operatorname{ent}\left(H_{1}\right)\right|\left|2-C e n t\left(H_{2}\right)\right| \\
& +\delta\left(H_{1}\right) \delta\left(H_{2}\right)\left|2-C \operatorname{ent}\left(H_{3}\right)\right| \\
& +\delta\left(H_{1}\right) \delta\left(H_{3}\right)|| 2-C \operatorname{Cent}\left(H_{2}\right) \mid \\
& +\delta\left(H_{2}\right) \delta\left(H_{3}\right)\left|2-C \operatorname{Cent}\left(H_{1}\right)\right| .
\end{aligned}
$$




\section{The Number of 2-Element Centralizers of $C A$-Groups}

The aim of this section is to compute the number of 2 -element centralizers of $C A$-groups. We start by the following crucial result.

Theorem 3.1. Let $G$ be a $C A$-group. Then the following holds:

(1) If $Z(G)=1$, then $|2-\operatorname{Cent}(G)|=|\operatorname{Cent}(G)|$,

(2) If $Z(G) \neq 1$, then $|2-\operatorname{Cent}(G)|=|\operatorname{Cent}(G)|+1$.

Proof. Since $G$ is a $C A$-group, by Theorem 1.2(15), for every $x, y \in G \backslash Z(G), C_{G}(x)=C_{G}(y)$ or $C_{G}(x) \cap C_{G}(y)=Z(G)$. Suppose $|\operatorname{Cent}(G)|=r$. Then there are $x_{1}, \ldots, x_{r-1} \in G \backslash Z(G)$ such that $\operatorname{Cent}(G)=\left\{G, C_{G}\left(x_{1}\right), \ldots, C_{G}\left(x_{r-1}\right)\right\}$. Consider the following two cases:

(1) $Z(G)=1$. It is clear that for every $1 \leq i \leq r-1, C_{G}\left(\left\{x_{i}, e\right\}\right)=C_{G}\left(x_{i}\right)$. By above argument $C_{G}\left(\left\{x_{i}, x_{j}\right\}\right)=C_{G}\left(x_{i}\right) \cap C_{G}\left(x_{j}\right)=Z(G)$, where $1 \leq i, j \leq r-1$ and $i \neq j$. Furthermore, there is no $x, y \in G$ such that $x \neq y$ and $G=C_{G}(x) \cap C_{G}(y)$. Therefore, $2-\operatorname{Cent}(G)=\left\{Z(G), C_{G}\left(x_{1}\right), \ldots, C_{G}\left(x_{r-1}\right)\right\}$ which implies that $|2-\operatorname{Cent}(G)|=|\operatorname{Cent}(G)|$.

(2) $Z(G) \neq 1$. It is clear that for every $x \in Z(G), C_{G}(\{x, e\})=C_{G}(x)=G$. By a similar argument as Part (1), $2-\operatorname{Cent}(G)=\left\{G, Z(G), C_{G}\left(x_{1}\right), \ldots, C_{G}\left(x_{r-1}\right)\right\}$. Thus, $|2-\operatorname{Cent}(G)|=$ $|\operatorname{Cent}(G)|+1$.

Hence the result.

Remark 3.2. Let $G$ be a centerless $C A$-group. Then $\mid$ Cent $(G)|=| 2-C e n t(G) \mid$ and so $G$ is primitive $n$ - and $(2, n)$-centralizer. Furthermore, if $G$ and $\frac{G}{Z}$ are both $C A$-group and $Z_{2}(G) \neq Z(G)$, then $G$ is primitive $n$-centralizer if and only if it is primitive $(2, n)$-centralizer.

Corollary 3.3. Let $G$ be a $C A$-group. $G$ is $(2, n)$-centralizer if and only if $G$ satisfies one of the following conditions:

(1) $Z(G)=1$ and $G$ is a n-centralizer.

(2) $Z(G) \neq 1$ and $G$ is a $(n-1)$-centralizer.

Proof. The result follows from Theorem 3.1

Theorem 3.4. Let $H$ be an abelian group, $p$ be a prime and $G=H \rtimes \mathbb{Z}_{p}$ is non-abelian. Then $|Z(G)||| H|,| \operatorname{Cent}(G) \mid=\frac{|H|}{|Z(G)|}+2$ and

$$
|2-\operatorname{Cent}(G)|= \begin{cases}|H|+2 & Z(G)=1 \\ \frac{|H|}{|Z(G)|}+3 & Z(G) \neq 1 .\end{cases}
$$

Proof. Since $G$ has an abelian normal subgroup of prime index, by Theorem $1.2(8),|C e n t(G)|=$ $\left|G^{\prime}\right|+2$. On the other hand, by Theorem 1.2(13), $|G|=p\left|G^{\prime}\right||Z(G)|$ and $\left|G^{\prime}\right|=\frac{|H|}{|Z(G)|}$. Hence, $|\operatorname{Cent}(G)|=\frac{|H|}{|Z(G)|}+2$. Moreover, since $G$ has an abelian normal subgroup of prime index, by Theorem 1.2(14), $G$ is a $C A$-group. We now apply Theorem 3.1 to deduce that

$$
|2-\operatorname{Cent}(G)|= \begin{cases}|H|+2 & Z(G)=1, \\ \frac{|H|}{|Z(G)|}+3 & Z(G) \neq 1,\end{cases}
$$

that completes the proof. 
Corollary 3.5. Suppose $\frac{G}{Z(G)} \cong \mathbb{Z}_{n} \rtimes \mathbb{Z}_{p}$ is non-abelian, $n$ is a positive integer and $p$ is a prime number. Then,

(1) If $Z(G)=1$, then $\left|\operatorname{Cent}\left(\mathbb{Z}_{n} \rtimes \mathbb{Z}_{p}\right)\right|=\left|2-\operatorname{Cent}\left(\mathbb{Z}_{n} \rtimes \mathbb{Z}_{p}\right)\right|=n+2$.

(2) Suppose $Z(G) \neq 1$.

(a) If $Z_{2}(G)=Z(G)$ then $|2-\operatorname{Cent}(G)|-1=|\operatorname{Cent}(G)|=\left|\operatorname{Cent}\left(\frac{G}{Z(G)}\right)\right|=\left|2-\operatorname{Cent}\left(\frac{G}{Z(G)}\right)\right|=$ $n+2$.

(b) If $Z_{2}(G) \neq Z(G)$ then $|2-\operatorname{Cent}(G)|-1=|\operatorname{Cent}(G)|=n+2$ and $\left|2-\operatorname{Cent}\left(\frac{G}{Z(G)}\right)\right|-1=$ $\left|\operatorname{Cent}\left(\frac{G}{Z(G)}\right)\right|=\frac{n}{\left|Z\left(\frac{G}{Z(G)}\right)\right|}+2$.

Proof. Since $\frac{G}{Z(G)} \cong \mathbb{Z}_{n} \rtimes \mathbb{Z}_{p}$, it has an abelian normal subgroup of prime index and so by Theorem 1.2(14), $\frac{G}{Z(G)}$ is a $C A$-group. Since $\frac{G}{Z(G)} \cong \mathbb{Z}_{n} \rtimes \mathbb{Z}_{p}$ is non-abelian and by Theorem 1.2(11), $G$ is a $C A$-group.

(1) Suppose $Z(G)=1$. By Remark $\left[3.2,\left|\operatorname{Cent}\left(\mathbb{Z}_{n} \rtimes \mathbb{Z}_{p}\right)\right|=\left|2-\operatorname{Cent}\left(\mathbb{Z}_{n} \rtimes \mathbb{Z}_{p}\right)\right|\right.$ and by Theorem $1.2(10),\left|\operatorname{Cent}\left(\mathbb{Z}_{n} \rtimes \mathbb{Z}_{p}\right)\right|=n+2$. Hence the result.

(2) Suppose $Z(G) \neq 1$.

(a) If $Z_{2}(G)=Z(G)$ then $Z\left(\frac{G}{Z(G)}\right)=1$. So by Theorems $\left[3.1(1)\right.$ and 3.4 , $\left|\operatorname{Cent}\left(\frac{G}{Z(G)}\right)\right|=$ $\left|2-\operatorname{Cent}\left(\frac{G}{Z(G)}\right)\right|=n+2$ and by Theorems $3.1(2)$ and $1.2(10),|2-\operatorname{Cent}(G)|-1=$ $|\operatorname{Cent}(G)|=n+2$.

(b) If $Z_{2}(G) \neq Z(G)$, then $Z\left(\frac{G}{Z(G)}\right) \neq 1$ and by Theorems $3.1(2)$ and $1.2(10),|2-C e n t(G)|-$ $1=|\operatorname{Cent}(G)|=n+2$. Now by Theorems $\left[3.1(2)\right.$ and $\left[3.4,\left|2-\operatorname{Cent}\left(\mathbb{Z}_{n} \rtimes \mathbb{Z}_{p}\right)\right|-1=\right.$ $\left|\operatorname{Cent}\left(\mathbb{Z}_{n} \rtimes \mathbb{Z}_{p}\right)\right|=\frac{n}{\left|Z\left(\mathbb{Z}_{n} \rtimes \mathbb{Z}_{p}\right)\right|}+2$.

This completes the proof.

Corollary 3.6. Let $G$ be a group such that $Z(G) \neq 1, \frac{G}{Z(G)} \cong \mathbb{Z}_{n} \rtimes \mathbb{Z}_{p}$ is non-abelian, $n$ is a positive integer and $p$ is prime. Then $G$ is primitive $n$-centralizer if and only if $Z_{2}(G)=Z(G)$.

Proof. If $Z_{2}(G)=Z(G)$, then by Corollary [3.5 (2a), $G$ is primitive $n$-centralizer. Conversely we assume that $G$ is primitive $n$-centralizer. Then $|\operatorname{Cent}(G)|=\left|\operatorname{Cent}\left(\frac{G}{Z(G)}\right)\right|$. On the other hand, by Theorems 3.4 and 1.2(10),$n+2=\frac{n}{\left|Z\left(\frac{G}{Z(G)}\right)\right|}+2$. Thus $\left|Z\left(\frac{G}{Z(G)}\right)\right|=1$ and so $Z_{2}(G)=Z(G)$.

Corollary 3.7. Let $G$ be a group with $\frac{G}{Z(G)} \cong \mathbb{Z}_{n} \rtimes \mathbb{Z}_{p}$ is non-abelian, $n$ is a positive integer and $p$ is prime. Then $G$ is primitive $(2, n)$-centralizer if and only if $Z(G)=1$.

Proof. If $Z(G)=1$, then obviously $G$ is primitive $(2, n)$-centralizer. Conversely, we assume that $G$ is a primitive $(2, n)$-centralizer. Hence $|2-\operatorname{Cent}(G)|=\left|2-\operatorname{Cent}\left(\frac{G}{Z(G)}\right)\right|$. Suppose $Z(G) \neq 1$. Then by Corollary 3.5 , both $G$ and $\frac{G}{Z}$ are $C A$-group. We now consider the following two cases:

(1) $Z\left(\frac{G}{Z(G)}\right)=1$. By Theorem 3.1 and Corollary $3.5(2 \mathrm{a}),\left|2-\operatorname{Cent}\left(\frac{G}{Z(G)}\right)\right|=\left|\operatorname{Cent}\left(\frac{G}{Z(G)}\right)\right|=n+2$. Since $Z(G) \neq 1$, by Theorems 3.1 and 1.2(10),,$|2-\operatorname{Cent}(G)|=|\operatorname{Cent}(G)|+1=n+3$ and by our assumption $|2-\operatorname{Cent}(G)|=\left|2-\operatorname{Cent}\left(\frac{G}{Z(G)}\right)\right|$. Thus, $n+3=n+2$, which is impossible.

(2) $Z\left(\frac{G}{Z(G)}\right) \neq 1$. By Theorem 3.1 and Corollary $\left[3.5(2 \mathrm{~b}),\left|2-\operatorname{Cent}\left(\frac{G}{Z(G)}\right)\right|-1=\left|\operatorname{Cent}\left(\frac{G}{Z(G)}\right)\right|=\right.$ $\frac{n}{\left|Z\left(\frac{G}{Z(G)}\right)\right|}+2$. Since $Z(G) \neq 1$, by Theorems 3.1 and $1.2(10),|2-\operatorname{Cent}(G)|=|\operatorname{Cent}(G)|+1=$ $n+3$. Now by our assumption, $|2-\operatorname{Cent}(G)|=\left|2-\operatorname{Cent}\left(\frac{G}{Z(G)}\right)\right|$. Thus, $n+3=\frac{n}{\left|Z\left(\frac{G}{Z(G)}\right)\right|}+3$ and so $Z\left(\frac{G}{Z(G)}\right)=1$ which is impossible. 
Hence $G$ is centerless and the proof is complete.

Theorem 3.8. Suppose $G$ is a finite non-abelian group and $r$ is the maximum size of a set of mutually non-commuting elements in $G$. Then $G$ is a $C A$-group if and only if

$$
|2-\operatorname{Cent}(G)|=\left\{\begin{array}{ll}
r+1 & Z(G)=1 \\
r+2 & Z(G) \neq 1
\end{array} .\right.
$$

Proof. Suppose $G$ is a $C A$-group. Then by Theorem 1.2(3), $|C e n t(G)|=r+1$. On the other hand, by Theorem 3.1 ,

$$
|2-\operatorname{Cent}(G)|= \begin{cases}|\operatorname{Cent}(G)| & Z(G)=1 \\ |\operatorname{Cent}(G)|+1 & Z(G) \neq 1\end{cases}
$$

and hence,

$$
|2-\operatorname{Cent}(G)|=\left\{\begin{array}{cc}
r+1 & Z(G)=1 \\
r+2 & Z(G) \neq 1
\end{array} .\right.
$$

Conversely, we assume that

$$
|2-\operatorname{Cent}(G)|=\left\{\begin{array}{cc}
r+1 & Z(G)=1 \\
r+2 & Z(G) \neq 1
\end{array} .\right.
$$

By Theorem 1.2(1),$r+1 \leq|\operatorname{Cent}(G)|$. We now consider the following cases:

(1) $Z(G)=1$. By Lemma $2.2(2)$ and our assumption $|\operatorname{Cent}(G)| \leq|2-\operatorname{Cent}(G)|=r+1$. Thus $|\operatorname{Cent}(G)|=r+1$ and by Theorem 1.2(3),$G$ is a $C A$-group.

(2) $Z(G) \neq 1$. By Lemma $2.2(1)$ and our assumption $|\operatorname{Cent}(G)|<|2-\operatorname{Cent}(G)|=r+2$. Hence $|\operatorname{Cent}(G)|=r+1$ and by Theorem 1.2(3),$G$ is a $C A$-group.

This completes our argument.

\section{Groups with at most Nine 2-Element Centralizers}

The aim of this section is to characterize finite groups with at most nine 2-element centralizers. One can easily seen that a group $G$ is $(2,1)$-centralizer if and only if $G$ is abelian which is similar to the case of $n$-centralizer groups. Also, there is no $(2,2)-$ and $(2,3)$-centralizer groups. In what follows it is also proved that there is no $(2,4)$-centralizer groups.

Theorem 4.1. There is no $(2,4)$-centralizer groups.

Proof. Suppose $G$ is a $(2,4)$-centralizer group. We will consider two cases as follows:

(1) $G$ is not centerless. By Lemma $2.2(1)$, $|\operatorname{Cent}(G)|<|2-\operatorname{Cent}(G)|=4$. So $|\operatorname{Cent}(G)| \leq 3$. On the other hand always $|\operatorname{Cent}(G)|>3$. This is impossible.

(2) $G$ is centerless. By Lemma $2.2(2)$, $\mid$ Cent $(G)|\leq| 2-\operatorname{Cent}(G) \mid=4$ and since $|\operatorname{Cent}(G)|>3$, $|\operatorname{Cent}(G)|=4$. Then by Theorem $1.1(1), G \cong \frac{G}{Z(G)} \cong \mathbb{Z}_{2} \times \mathbb{Z}_{2}$, a contradiction.

Hence there is no $(2,4)$-centralizer groups.

Lemma 4.2. If $|\operatorname{Cent}(G)|=6$ and $\frac{G}{Z(G)} \cong \mathbb{Z}_{2} \times \mathbb{Z}_{2} \times \mathbb{Z}_{2} \times \mathbb{Z}_{2}$. Then $G$ is a $C A$-group. 
Proof. Suppose $\left\{x_{1}, \ldots, x_{r}\right\}$ is a set of pairwise non-abelian elements of $G$ with maximal size. So, by Theorem 1.2(1b), $r+1 \leq|\operatorname{Cent}(G)|=6$. Thus $r \leq 5$. On the other hand, by Theorem 1.2(1a), $r \geq 3$. This shows that $3 \leq r \leq 5$. If $r=3$, then by $1.2(1 \mathrm{c})$ we have $|\operatorname{Cent}(G)|=4$, which is a contradiction. If $r=4$, then by $1.2(1 \mathrm{~d}),|\operatorname{Cent}(G)|=5$ which leads to another contradiction. Therefore, $r=5$ and $\operatorname{Cent}(G)=\left\{G, C_{G}\left(x_{1}\right), \ldots, C_{G}\left(x_{5}\right)\right\}$. Since $6=|\operatorname{Cent}(G)|<r+4=9$, by Theorem 1.2(2a) we have $C_{G}(x)$ is abelian, where $x \in G \backslash Z(G)$ is arbitrary. Thus $G$ is a $C A$-group, as desired.

Corollary 4.3. Suppose $G$ is an $n$-centralizer with $n \leq 9$. Then $G$ is a $C A$-group.

Proof. The proof follows from Theorems 1.1, 1.2(6) and Lemma 4.2 .

Lemma 4.4. Suppose $G$ is a $(2, n)$-centralizer group with $n \leq 9$. Then $G$ is a $C A$-group.

Proof. Let $G$ be a $(2, n)$-centralizer and $n \leq 9$. If $Z(G) \neq 1$, then by Lemma $2.2(1)$, $\mid$ Cent $(G) \mid<$ $|2-\operatorname{Cent}(G)|=n \leq 9$. So by Corollary 4.3, $G$ is a $C A$-group. If $Z(G)=1$ then by Lemma $2.2(2)$, $|\operatorname{Cent}(G)| \leq|2-\operatorname{Cent}(G)|=n \leq 9$. So by Corollary 4.3, $G$ is a $C A$-group.

Theorem 4.5. A group $G$ is $(2,5)$-centralizer if and only if $G \cong S_{3}$ or $G$ is not centerless and $\frac{G}{Z(G)} \cong \mathbb{Z}_{2} \times \mathbb{Z}_{2}$. Moreover, $G$ is primitive $(2,5)$-centralizer if and only if $G \cong S_{3}$.

Proof. Suppose $G$ is $(2,5)$-centralizer. By Lemma 4.4, $G$ is a $C A$-group and by Corollary 3.3, the following two cases can be occurred:

(1) $Z(G)=1$ and $G$ is 5 -centralizer. By Theorem $1.1(2), G \cong \frac{G}{Z(G)} \cong \mathbb{Z}_{3} \times \mathbb{Z}_{3}$ or $S_{3}$ and since $G$ is non-abelian, $G \cong S_{3}$.

(2) $Z(G) \neq 1$ and $G$ is 4 -centralizer. By Theorem $1.1(1), \frac{G}{Z(G)} \cong \mathbb{Z}_{2} \times \mathbb{Z}_{2}$, as desired.

In order to prove the converse of this theorem, we note that $S_{3}$ is obviously $(2,5)$-centralizer. We assume that $\frac{G}{Z(G)} \cong \mathbb{Z}_{2} \times \mathbb{Z}_{2}$. Clearly $G$ can not be centerless and by Theorem $[1.1(1), \mid$ Cent $(G) \mid=4$. By Theorem 1.2(6),$G$ is a $C A$-group and by Theorem 3.1, $|2-\operatorname{Cent}(G)|=|\operatorname{Cent}(G)|+1=5$. This proves that $G$ is a $(2,5)$-centralizer group.

If $G$ is primitive $(2,5)$-centralizer, then $|2-\operatorname{Cent}(G)|=\left|2-\operatorname{Cent}\left(\frac{G}{Z(G)}\right)\right|=5$. By the first part of this theorem, $G \cong S_{3}$ or $\frac{G}{Z(G)} \cong \mathbb{Z}_{2} \times \mathbb{Z}_{2}$. Since $\mathbb{Z}_{2} \times \mathbb{Z}_{2}$ is abelian, $\left|2-\operatorname{Cent}\left(\frac{G}{Z(G)}\right)\right|=1$ which is a contradiction. Thus $G \cong S_{3}$, as desired.

Theorem 4.6. A group $G$ is $(2,6)$-centralizer if and only if $G \cong A_{4}$ or $G$ is not centerless and $\frac{G}{Z(G)} \cong \mathbb{Z}_{3} \times \mathbb{Z}_{3}$ or $S_{3}$. Moreover, $G$ is primitive $(2,6)$-centralizer if and only if $G \cong A_{4}$.

Proof. Suppose $G$ is $(2,6)$-centralizer. By Lemma 4.4, $G$ is a $C A$-group and by Corollary 3.3, the following two cases can be occurred:

(1) $G$ is centerless and $G$ is a 6 -centralizer. By Theorem $1.1(3), G \cong \frac{G}{Z(G)} \cong \mathbb{Z}_{2} \times \mathbb{Z}_{2} \times \mathbb{Z}_{2}, \mathbb{Z}_{2} \times$ $\mathbb{Z}_{2} \times \mathbb{Z}_{2} \times \mathbb{Z}_{2}, D_{8}$ or $A_{4}$. Since $G$ is a non-abelian group and $\left|2-\operatorname{Cent}\left(D_{8}\right)\right|=5, G \cong A_{4}$.

(2) $G$ is not centerless and $G$ is 5 -centralizer. By Theorem $1.1(2), \frac{G}{Z(G)} \cong \mathbb{Z}_{3} \times \mathbb{Z}_{3}$ or $S_{3}$, as desired.

Conversely, if $G \cong A_{4}$ then $\left|2-\operatorname{Cent}\left(A_{4}\right)\right|=6$. It is enough to assume that $G$ is not centerless and $\frac{G}{Z(G)} \cong \mathbb{Z}_{3} \times \mathbb{Z}_{3}$ or $S_{3}$. By Theorem $1.1(2),|C e n t(G)|=5$ and by Theorem [1.2(6), $G$ is a $C A$-group. Moreover, by Theorem $[3.1(2),|2-\operatorname{Cent}(G)|=|\operatorname{Cent}(G)|+1=6$. This proves that $G$ is $(2,6)$-centralizer. 
If $G$ is a primitive $(2,6)$-centralizer, then $|2-\operatorname{Cent}(G)|=\left|2-\operatorname{Cent}\left(\frac{G}{Z(G)}\right)\right|=6$. By the first part of this theorem, $G \cong A_{4}$ or $G$ is not centerless and so $\frac{G}{Z(G)} \cong \mathbb{Z}_{3} \times \mathbb{Z}_{3}$ or $S_{3}$. Since $\left|2-\operatorname{Cent}\left(\mathbb{Z}_{3} \times \mathbb{Z}_{3}\right)\right|=1$ and $\left|2-\operatorname{Cent}\left(S_{3}\right)\right|=5, G \cong A_{4}$ which completes our argument.

Theorem 4.7. A group $G$ is $(2,7)$-centralizer if and only if $G \cong D_{10}, R$ or $G$ is not centerless and it is 6 -centralizer. Moreover, $G$ is primitive $(2,7)$-centralizer if and only if $G \cong D_{10}$ or $R$.

Proof. Suppose $G$ is $(2,7)$-centralizer. By Lemma 4.4, $G$ is a $C A$-group and by Corollary 3.3, $G$ is a 6-centralizer group with non-trivial center or a centerless 7 -centralizer group. The first case leads to our result and in the second case, $G \cong D_{10}$ or $R$, as desired.

Conversely, if $G \cong D_{10}$ or $R$, then $\left|2-\operatorname{Cent}\left(D_{10}\right)\right|=|2-\operatorname{Cent}(R)|=7$, as desired. So, it is enough to assume that $G$ is not centerless and 6-centralizer. By Theorem $1.1(3), \frac{G}{Z(G)} \cong \mathbb{Z}_{2} \times \mathbb{Z}_{2} \times \mathbb{Z}_{2}, \mathbb{Z}_{2} \times$ $\mathbb{Z}_{2} \times \mathbb{Z}_{2} \times \mathbb{Z}_{2}, D_{8}$ or $A_{4}$. By Theorem 1.2(6) and Lemma 4.2, $G$ is $C A$-group and by Theorem $3.1(2)$, $|2-\operatorname{Cent}(G)|=|\operatorname{Cent}(G)|+1=7$. This proves that $G$ is $(2,7)$-centralizer.

If $G$ is primitive $(2,7)-$ centralizer. Then $|2-\operatorname{Cent}(G)|=\left|2-\operatorname{Cent}\left(\frac{G}{Z(G)}\right)\right|=7$. By the first part of this theorem, $G \cong D_{10}, R$ or $G$ is not centerless and 6 -centralizer. Since $\left|2-\operatorname{Cent}\left(\mathbb{Z}_{2} \times \mathbb{Z}_{2} \times \mathbb{Z}_{2}\right)\right|=$ $\left|2-\operatorname{Cent}\left(\mathbb{Z}_{2} \times \mathbb{Z}_{2} \times \mathbb{Z}_{2} \times \mathbb{Z}_{2}\right)\right|=1,\left|2-\operatorname{Cent}\left(D_{8}\right)\right|=5$ and $\left|2-\operatorname{Cent}\left(A_{4}\right)\right|=6$. So $G \cong D_{10}, R$ which completes our argument.

Theorem 4.8. A group $G$ is $(2,8)$-centralizer if and only if $G$ is a 7 -centralizer group with nontrivial center. Moreover, There is no primitive $(2,8)$-centralizer group.

Proof. Suppose $G$ is $(2,8)$-centralizer. Apply Lemma 4.4 to deduce that $G$ is a $C A$-group. By Corollary [3.3, $G$ is a centerless 8 -centralizer group or a 7 -centralize group with non-trivial center. If $G$ is a centerless 8 -centralizer group then by Theorem $1.1(6), G \cong \frac{G}{Z(G)} \cong \mathbb{Z}_{2} \times \mathbb{Z}_{2} \times \mathbb{Z}_{2}, D_{12}$ or $A_{4}$. But $G$ is a non-abelian group and $\left|2-\operatorname{Cent}\left(D_{12}\right)\right|=\left|2-\operatorname{Cent}\left(A_{4}\right)\right|=6$, which is impossible. Therefore, $G$ is a 7 -centralizer group with non-trivial center, as desired.

Conversely, suppose $G$ is not centerless and it is a 7 -centralizer group. Then, by Theorem 1.1(5), $\frac{G}{Z(G)} \cong \mathbb{Z}_{5} \times \mathbb{Z}_{5}, D_{10}$ or $R$, and by Theorem 1.2(6),$G$ is $C A$-group. We now apply Theorem $3.1(2)$ to deduce that $|2-\operatorname{Cent}(G)|=|\operatorname{Cent}(G)|+1=8$. This proves that $G$ is $(2,8)-$ centralizer.

If $G$ is primitive $(2,8)$-centralizer. Then $|2-\operatorname{Cent}(G)|=\left|2-\operatorname{Cent}\left(\frac{G}{Z(G)}\right)\right|=8$. By the first part of this theorem, $G$ is a 7 -centralizer group with non-trivial center and by Theorem 1.1(5), $\frac{G}{Z(G)} \cong \mathbb{Z}_{5} \times \mathbb{Z}_{5}, D_{10}$ or $R$. Since $\left|2-\operatorname{Cent}\left(\mathbb{Z}_{5} \times \mathbb{Z}_{5}\right)\right|=1$ and $\left|2-\operatorname{Cent}\left(D_{10}\right)\right|=|2-\operatorname{Cent}(R)|=7$. So, there is no primitive $(2,8)$-centralizer group which completes our argument.

Theorem 4.9. A group $G$ is (2,9)-centralizer if and only if $G \cong D_{14}, \operatorname{Hol}\left(\mathbb{Z}_{7}\right)$, a non-abelian group of order 21 or $G$ is a 8-centralizer group with non-trivial center. Moreover, $G$ is primitive $(2,9)$-centralizer if and only if $G \cong D_{14}, H o l\left(\mathbb{Z}_{7}\right)$ or a non-abelian group of order 21 .

Proof. Suppose $G$ is $(2,9)$-centralizer. Then, by Lemma 4.4, $G$ is a $C A$-group and by Corollary 3.3 , $G$ is a 8 -centralizer group with non-trivial center or $G$ is a centerless 9 -centralizer group. In later, we apply Theorem $1.1(7)$ to deduce that $G \cong \frac{G}{Z(G)} \cong \mathbb{Z}_{7} \times \mathbb{Z}_{7}, D_{14}, \operatorname{Hol}\left(\mathbb{Z}_{7}\right)$ or a non-abelian group of order 21. Since $G$ is a non-abelian group, $G \cong D_{14}, \operatorname{Hol}\left(\mathbb{Z}_{7}\right)$ or a non-abelian group of order 21 , as desired.

Conversely, if $G \cong D_{14}, \operatorname{Hol}\left(\mathbb{Z}_{7}\right)$ or a non-abelian group $L$ of order 21 , then $\left|2-\operatorname{Cent}\left(D_{14}\right)\right|=$ $\left|2-\operatorname{Cent}\left(\operatorname{Hol}\left(\mathbb{Z}_{7}\right)\right)\right|=|2-\operatorname{Cent}(L)|=9$, as desired. So, it is enough to assume that $G$ is a 8 -centralizer 
group with non-trivial center. By Theorem $1.1(6), \frac{G}{Z(G)} \cong \mathbb{Z}_{2} \times \mathbb{Z}_{2} \times \mathbb{Z}_{2}, D_{12}$ or $A_{4}$, and by Theorem 1.2(6), $G$ is $C A$-group. We now apply Theorem 3.1 (2) to deduce that $|2-\operatorname{Cent}(G)|=|\operatorname{Cent}(G)|+1=$ 9. This proves that $G$ is $(2,9)$-centralizer.

If $G$ is primitive $(2,9)$-centralizer, then $|2-\operatorname{Cent}(G)|=\left|2-\operatorname{Cent}\left(\frac{G}{Z(G)}\right)\right|=9$. By the first part of theorem, $G \cong D_{14}, \operatorname{Hol}\left(\mathbb{Z}_{7}\right)$, a non-abelian group of order 21 or $G$ a 8 -centralizer group with non-trivial center. Since $\left|2-\operatorname{Cent}\left(\mathbb{Z}_{2} \times \mathbb{Z}_{2} \times \mathbb{Z}_{2}\right)\right|=1$ and $\left|2-\operatorname{Cent}\left(D_{12}\right)\right|=\left|2-\operatorname{Cent}\left(A_{4}\right)\right|=6$, $G \cong D_{14}, \mathrm{Hol}\left(\mathbb{Z}_{7}\right)$ or a non-abelian group of order 21 . This completes our argument.

\section{Finite Groups with a Given Number of 2-Eleemnt Centralizers}

In this section, a characterization of the alternating group $A_{5}$ with respect to the number of 2 -element centralizers is given. We also prove that all finite groups with at most 212 -element centralizers are solvable.

Lemma 5.1. Let $G$ be a finite group such that $\frac{G}{Z(G)} \cong A_{5}$. Then $G \cong A_{5}$ or $|2-\operatorname{Cent}(G)|=$ $|\operatorname{Cent}(G)|+1=23,33$.

Proof. It is easy to see that all element centralizers of $A_{5}$ are Sylow subgroups of $A_{5}$ and so each pair of them have trivial intersection. If $Z(G)=1$ then $G \cong A_{5}$. Suppose $Z=Z(G) \neq 1$, then $G$ is not abelian and by Lemma 2.1 $(2), G \in 2-\operatorname{Cent}(G)$. Choose arbitrary elements $x, y \in G$. Obviously $\frac{C_{G}(x)}{Z} \leqslant C_{G / Z}(x Z)$ and $\frac{C_{G}(y)}{Z} \leqslant C_{G / Z}(y Z)$. Hence $\frac{C_{G}(x)}{Z} \cap \frac{C_{G}(y)}{Z} \leqslant C_{G / Z}(x Z) \cap C_{G / Z}(y Z)=1_{G / Z}$. Therefore $\frac{C_{G}(x) \cap C_{G}(y)}{Z}=1$ and so $C_{G}(x) \cap C_{G}(y)=Z$. Thus, $Z \in 2-\operatorname{Cent}(G)$ which proves that $|2-\operatorname{Cent}(G)|=|\operatorname{Cent}(G)|+1$. Finally, Theorem [1.2(4) implies that $|2-\operatorname{Cent}(G)|=23$ or 33 .

Theorem 5.2. Let $G$ be a finite group. The following are hold:

(1) If $|2-\operatorname{Cent}(G)|<22$, then $G$ is solvable.

(2) If $G$ is simple and $|2-\operatorname{Cent}(G)|=22$, then $G \cong A_{5}$.

Proof. Suppose $|2-\operatorname{Cent}(G)|<22$. By Lemma 2.2. $|\operatorname{Cent}(G)| \leq|2-\operatorname{Cent}(G)|<22$ and so by Theorem 1.2(16), $G$ is solvable. This proves part (1). We now assume that $G$ is simple and $\mid 2-$ $\operatorname{Cent}(G) \mid=22$. Again by Lemma 2.2, $|\operatorname{Cent}(G)| \leq 22$. If $|\operatorname{Cent}(G)| \leq 21$, then by Theorem 1.2(16), $G$ is solvable, contradicts by simplicity of $G$. Therefore, $|\operatorname{Cent}(G)|=22$ and by Theorem $1.2(5)$, $G \cong A_{5}$.

Remark 5.3. Suppose $G$ is a finite non-abelian simple group with $|2-\operatorname{Cent}(G)| \leq 100$. Then by Lemma 2.2(2), $|\operatorname{Cent}(G)| \leq|2-\operatorname{Cent}(G)| \leq 100$ and by [11, Theorem A], the group $G$ is isomorphic to one of the simple groups $\operatorname{PSL}(2,5), \operatorname{PSL}(2,7)$ or $\operatorname{PSL}(2,8)$.

Our calculations with the aid of Gap suggest the following conjecture:

Conjecture 5.4. Suppose $G$ and $H$ are finite simple group and $|2-\operatorname{Cent}(G)|=|2-\operatorname{Cent}(H)|$. Then $G \cong H$.

Theorem 5.5. Let $G$ be a group with center $Z$ such that $[G: Z]=p^{n}, p$ is prime. Moreover, we assume that the order all proper centralizers of $G$ are equal to $p|Z|$. Then,

$$
\begin{aligned}
|\operatorname{Cent}(G)| & =p^{n-1}+p^{n-2}+\cdots+p+2, \\
|2-\operatorname{Cent}(G)| & =\underset{12}{|\operatorname{Cent}(G)|+1 .}
\end{aligned}
$$


Proof. Since $G$ is non-abelian and $Z \neq 1$, Lemma 2.1(2) implies that $G \in 2-\operatorname{Cent}(G)$. We claim that for each $x, y \in G \backslash Z, C_{G}(x)=C_{G}(y)$ or $C_{G}(x) \cap C_{G}(y)=Z$. To prove, we assume that $C_{G}(x) \cap C_{G}(y) \neq$ $Z$. Thus, $Z \supsetneqq C_{G}(x) \cap C_{G}(y) \leq C_{G}(x)$. Since $\left|C_{G}(x): Z\right|=p, C_{G}(x) \cap C_{G}(y)=C_{G}(x)=C_{G}(y)$. This shows that $C_{G}(x)=C_{G}(y)$. Hence $|2-\operatorname{Cent}(G)|=|\operatorname{Cent}(G)|+1$. Suppose $m$ is the number of distinct proper centralizer of $G$. Then $|G|-|Z|=m\left(\left|C_{G}(x)\right|-|Z|\right)$ and so $\left(p^{n}-1\right)|Z|=m(p-1)|Z|$. Therefore, $m=\frac{p^{n}-1}{p-1}=p^{n-1}+p^{n-2}+\cdots+p+1$. Thus $|\operatorname{Cent}(G)|=p^{n-1}+p^{n-2}+\cdots+p+2$. This completes the proof.

Theorem 5.6. Let $p$ be a prime number and $G$ be a group with center $Z$ such that $\frac{G}{Z}=\mathbb{Z}_{p} \times \cdots \times \mathbb{Z}_{p}$. If all proper centralizers of $G$ are of order $p|Z|$ or $p^{2}|Z|$. Then,

$$
\begin{aligned}
|\operatorname{Cent}(G)| & =s+t+1, \\
|2-\operatorname{Cent}(G)| & =|\operatorname{Cent}(G)|+1,
\end{aligned}
$$

where $s$ and $t$ are the number of distinct centralizers of $G$ of orders $p|Z|$ and $p^{2}|Z|$, respectively. Moreover, $s+t(p+1)=p^{n-1}+p^{n-2}+\cdots+p+1$.

Proof. Since $G$ is a non-abelian group and $Z$ is not a trivial subgroup, Lemma 2.1(2) implies that $G \in 2-\operatorname{Cent}(G)$. It is clear that for every $x \in G \backslash Z, x^{p} \in Z$. In what follow, two cases that $\left|C_{G}(x)\right|=p|Z|$ and $\left|C_{G}(x)\right|=p^{2}|Z|$ are considered separately.

(1) $\left|C_{G}(x)\right|=p|Z|$. In this case, $A=\left\{x^{i} z \mid 0 \leq i \leq p-1 \& z \in Z\right\} \subseteq C_{G}(x)$ and since $|A|=p|Z|$, $C_{G}(x)=A$. It is clear that $C_{G}(x) \subseteq C_{G}\left(x^{i}\right), 1 \leq i \leq p-1$. We prove that under the condition that $1 \leq i \leq p-1, C_{G}(x)=C_{G}\left(x^{i}\right)$. Since $(i, p)=1$, there exists $m$ and $k$ such that $m i+k p=1$. Suppose $y \in C_{G}\left(x^{i}\right)$. Then, $y\left(x^{i}\right)^{m}=\left(x^{i}\right)^{m} y$ and so $y x^{1-k p}=x^{1-k p} y$. Since $x^{p} \in Z, y x=x y$ which implies that $y \in C_{G}(x)$. Therefore,

$$
C_{G}\left(x^{i}\right)=C_{G}(x), 1 \leq i \leq p-1 .
$$

(2) If $\left|C_{G}(x)\right|=p^{2}|Z|$. In this case, $A=\left\{x^{i} z \mid 0 \leq i \leq p-1 \& z \in Z\right\} \subseteq C_{G}(x)$ and since $|A|=p|Z|, A \subset C_{G}(x)$. Choose $y \in C_{G}(x) \backslash A$. Then $B=\left\{x^{i} y^{j} z \mid 0 \leq i, j \leq p-1\right\} \subseteq C_{G}(x)$. Note that $|B|=p^{2}|Z|$ and so $C_{G}(x)=B$. Since $y \in C_{G}(x), C_{G}(x) \subseteq C_{G}\left(x^{i} y^{j}\right), 1 \leq i, j \leq p-1$. By assumption and our last inclusion, $p^{2}|Z|=\left|C_{G}(x)\right| \leq\left|C_{G}\left(x^{i} y^{j}\right)\right| \leq p^{2}|Z|$. Therefore,

$$
C_{G}\left(x^{i} y^{j}\right)=C_{G}(x), 1 \leq i, j \leq p-1 .
$$

We claim that for every $x, y \in G \backslash Z$, one of the following hold:

(1) If $\left|C_{G}(x)\right|=\left|C_{G}(y)\right|$, then $C_{G}(x)=C_{G}(y)$ or $C_{G}(x) \cap C_{G}(y)=Z$.

(2) If $\left|C_{G}(x)\right| \neq\left|C_{G}(y)\right|$, then $C_{G}(x) \cap C_{G}(y)=Z$.

Suppose $C_{G}(x) \cap C_{G}(y) \neq Z$. Then there exists $u \in C_{G}(x) \cap C_{G}(y) \backslash Z$. Thus $u \in C_{G}(x) \backslash Z$ and $u \in C_{G}(y) \backslash Z$. We now apply Equations 5.1 and 5.2 to deduce that $C_{G}(x)=C_{G}(u)=C_{G}(y)$. This completes the proof of Parts (1) and (2).

Our above discussion show that $|2-\operatorname{Cent}(G)|=|\operatorname{Cent}(G)|+1$. Suppose the number of distinct centralizers of $G$ of orders $p|Z|$ and $p^{2}|Z|$ are $s$ and $t$, respectively. Therefore, $s\left(\left|C_{G}(x)\right|-|Z|\right)+$ $t\left(\left|C_{G}(y)\right|-|Z|\right)=|G|-|Z|$ and so $s(p-1)|Z|+t(p-1)(p+1)|Z|=\left(p^{n}-1\right)|Z|$. This proves that $s+t(p+1)=p^{n-1}+p^{n-2}+\cdots+p+1$ which our argument. 


\section{ExAmples}

The aim of this section is to apply our results in Sections 2-5 for computing the number of $(2, n)$-centralizers in certain finite groups. We start by non-abelian $p$-groups of order $p^{4}$.

Example 6.1. In this example we calculate the number of centralizers and 2-element centralizers of a non-abelian $p$-group of order $p^{4}$. It is proved that $|\operatorname{Cent}(G)|=p+2, p^{2}+2$ or $p^{2}+p+2$ and in any case $|2-\operatorname{Cent}(G)|=|\operatorname{Cent}(G)|+1$. Since $G$ is non-abelian, $|Z(G)|=p$ or $p^{2}$.

(1) $|Z(G)|=p$. By Theorem 1.2(7), $\mid$ Cent $(G) \mid=p^{2}+2$ or $p^{2}+p+2$. Since $|G: Z(G)|=p^{3}$, by Theorem 1.2(6),$G$ is a $C A$-group and by Theorem [3.1(2), $|2-\operatorname{Cent}(G)|=|\operatorname{Cent}(G)|+1$.

(2) $|Z(G)|=p^{2}$. In this case, $\frac{G}{Z(G)} \cong \mathbb{Z}_{p} \times \mathbb{Z}_{p}$ and by Theorem 1.2(12), $|\operatorname{Cent}(G)|=p+2$. On the other hand, since $G$ is a group of order $p^{4}$ and $|Z(G)|=p^{2}, \frac{G}{Z(G)} \cong Z_{p} \times Z_{p}$. Therefore, by Theorem 5.6, $|2-\operatorname{Cent}(G)|=|\operatorname{Cent}(G)|+1$.

Example 6.2. In this example, the number of 2-element centralizers of a finite group $G$ with this property that $\frac{G}{Z(G)} \cong D_{2 n}$ is computed, where $n \geq 3$ is a positive integer. By Corollary [3.5, one can easily seen that

(1) If $Z(G)=1$, then $\left|\operatorname{Cent}\left(D_{2 n}\right)\right|=\left|2-\operatorname{Cent}\left(D_{2 n}\right)\right|=n+2$.

(2) If $Z(G) \neq 1$ then,

(a) If $n$ is odd. Then $|2-\operatorname{Cent}(G)|-1=|\operatorname{Cent}(G)|=\left|\operatorname{Cent}\left(D_{2 n}\right)\right|=\left|2-\operatorname{Cent}\left(D_{2 n}\right)\right|=n+2$.

(b) If $n$ is even. Then $|2-\operatorname{Cent}(G)|-1=|\operatorname{Cent}(G)|=n+2$ and $\left|2-\operatorname{Cent}\left(D_{2 n}\right)\right|-1=$ $\left|\operatorname{Cent}\left(D_{2 n}\right)\right|=\frac{n}{2}+2$.

Example 6.3. The semi-dihedral group $S D_{8 n}$ can be presented as $\left\langle a, b \mid a^{4 n}=b^{2}=e, b a b=a^{2 n-1}\right\rangle$, where $n \geq 2$ is a positive integer. By Corollary [3.5,

$$
\left|2-\operatorname{Cent}\left(S D_{8 n}\right)\right|=\left|\operatorname{Cent}\left(S D_{8 n}\right)\right|+1=\left\{\begin{array}{ll}
n+3 & n \text { is odd } \\
2 n+3 & n \text { is even }
\end{array} .\right.
$$

Example 6.4. The dicyclic group $T_{4 n}$ can be presented as $\left\langle a, b \mid a^{2 n}=e, a^{n}=b^{2}, b^{-1} a b=a^{-1}\right\rangle$, where $n \geq 2$ is a positive integer. Since $\frac{T_{4 n}}{Z\left(T_{4 n}\right)} \cong D_{2 n}=Z_{n} \rtimes Z_{2}$, by Corollary [3.5, we have $\left|2-\operatorname{Cent}\left(T_{4 n}\right)\right|=$ $\left|\operatorname{Cent}\left(T_{4 n}\right)\right|+1=n+3$.

Example 6.5. The group $V_{8 n}$ can be presented as $\left\langle a, b \mid a^{2 n}=b^{4}=e, a b a=b^{-1}, a b^{-1} a=b\right\rangle$, where $n$ is a positive integer. Note that

$$
\frac{V_{8 n}}{Z} \cong \begin{cases}Z_{2 n} \rtimes Z_{2} & 2 \nmid n \\ Z_{n} \rtimes Z_{2} & 2 \mid n\end{cases}
$$

Then by Corollary 3.5, we have

$$
\left|2-\operatorname{Cent}\left(V_{8 n}\right)\right|=\left|\operatorname{Cent}\left(V_{8 n}\right)\right|+1=\left\{\begin{array}{ll}
2 n+3 & n \text { is odd } \\
n+3 & n \text { is even }
\end{array} .\right.
$$

Example 6.6. The group $U_{2(n, m)}$ can be presented as $U_{2(n, m)}=\left\langle a, b \mid a^{2 n}=b^{m}=e, a b a^{-1}=b^{-1}\right\rangle$. If $m=1,2$. Then $U_{2(n, m)}$ is an abelian group and so $\left|2-\operatorname{Cent}\left(U_{2(m, n)}\right)\right|=\left|\operatorname{Cent}\left(U_{2(m, n)}\right)\right|=1$. In 
other cases, $\frac{U_{2(m, n)}}{Z} \cong Z_{m} \rtimes Z_{2}$ and by Corollary 3.5,

$$
\left|2-\operatorname{Cent}\left(U_{2(m, n)}\right)\right|=\left\{\begin{array}{ll}
\left|\operatorname{Cent}\left(U_{2(m, n)}\right)\right|=m+2 & m \text { is odd and } n=1 \\
\left|\operatorname{Cent}\left(U_{2(m, n)}\right)\right|+1=m+3 & m \text { is odd and } n \neq 1 \\
\left|\operatorname{Cent}\left(U_{2(m, n)}\right)\right|+1=\frac{m}{2}+3 & m \text { is even }
\end{array} .\right.
$$

Acknowledgement. The research of the authors are partially supported by the University of Kashan under grant no $785149 / 70$.

\section{REFERENCES}

[1] A. Abdollahi, S. M. Jafarian Amiri and A. Mohammadi Hassanabadi, Groups with specific number of centralizers, Houston J. Math. 33 (1) (2007) 43-57.

[2] A. R. Ashrafi, On finite groups with a given number of centralizers, Algebra Colloq. 7 (2000) 139-146.

[3] A. R. Ashrafi and B. Taeri, On finite groups with exactly seven element centralizers, J. Appl. Math. Comput. 22 (1-2) (2006) 403-410.

[4] A. R. Ashrafi and B. Taeri, On finite groups with a certain number of centralizers, J. Appl. Math. Comput. 17 (1-2) (2005) 217-227.

[5] S. J. Baishya, On finite groups with nine centralizers, Boll. Unione Mat. Ital. 9 (2016) 527-531.

[6] S. J. Baishya, On finite groups with specific number of centralizers, Int. Electron. J. Alg. 13 (2013) 53-62.

[7] S. M. Belcastro and G. J. Sherman, Counting centralizers in finite groups, Math. Mag. 5 (1994) $111-114$.

[8] Ya. G. Berkovich and E. M. Zhmud', Characters of Finite Groups. Part 1, Translated from the Russian manuscript by P. Shumyatsky [P. V. Shumyatski] and V. Zobina. Translations of Mathematical Monographs, 172. American Mathematical Society, Providence, RI, 1998.

[9] S. Dolfi, M. Herzog and E. Jabara, Finite groups whose noncentral commuting elements have centralizers of equal size, Bull. Aust. Math Soc. 82 (2010) 293-304.

[10] Z. Foruzanfar and Z. Mostaghim, On 10-centralizer groups of odd order, ISRN Algebra 2014 Article ID 607984, 4 pages.

[11] Z. Foruzanfar, Z. Mostaghim and M. Rezaei, Non-abelian finite simple groups with a bounded number of centralizers, 23th Iranian Algebra Seminar, Faculty of Computer and Mathematics, Khansar, Iran, November 21-22, 2013.

[12] N. Itô, On finite groups with given conjugate types I, Nagoya Math. J. 6 (1953) 17-28.

[13] S. M. Jafarian Amiri, H. Madadi and H. Rostami, On 9-centralizer groups, J. Algebra Appl. 14 (1) (2015) 1550003 (13 pages).

[14] R. D. Kitture, Groups with finitely many centralizers, Bull. Allahabad Math. Soc. 30 (2015) $29-37$.

[15] M. Rezaei and Z. Foruzanfar, On primitive 11-centralizer groups of odd order, Malays. J. Math. Sci. 10 (3) (2016) 361-368.

[16] D. J. Robinson, A Course in the Theory of Groups, Springer-Verlag, New York, 1996.

[17] R. Schmidt, Zentralisatorverbände endlicher Gruppen, Rend. Sem. Mat. Univ. Padova 44 (1970) $97-131$.

[18] The GAP Team, Group, GAP - Groups, Algorithms, and Programming, Version 4.5.5, 2012, http://www.gapsystem.org.

[19] M. Zarrin, On element-centralizers in finite groups, Arch. Math. 93 (2009) 497-503.

[20] M. Zarrin, On solubility of groups with finitely many centralizers, Bull. Iranian Math. Soc. 39 (3) (2013) $517-521$.

Department of Pure Mathematics, Faculty of Mathematical Sciences, University of Kashan, Kashan 87317-53153, I. R. IRAN 